\title{
Lupus eritematoso sistemico all'esordio complicato da un severo coinvolgimento multiorgano
}

\author{
Silvia Forcellini, Mariangela Annaloro, Alessia Bortot, Giorgia Russo, Yuri Battaglia \\ U.O.C Nefrologia, Azienda Ospedaliero Universitaria Sant'Anna Cona, Ferrara
}

\begin{abstract}
ONSET OF SYSTEMIC LUPUS ERYTHEMATOUS COMPLICATED BY SEVERE MULTIORGAN INVOLVEMENT
Abstract. The authors report the case of a 51-year-old man with a 1-month history of mild gastroenteritic symptoms, daily fever and malaise. The patient was febrile $\left(38.5^{\circ} \mathrm{C}\right.$ ) and laboratory data showed renal failure (creatinine $2.5 \mathrm{mg} / \mathrm{dL}$ ), proteinuria and hemoglobinuria. A 10-day antibiotic therapy was started since an infective gastrointestinal process was initially suspected. When autoimmune immunologic patterns, associated with renal, neurologic and hematologic signs, confirmed the diagnosis of systemic lupus erythematous the patient was infused with high-dose intravenous corticosteroids. Despite the treatment, the patient developed rhabdomyolysis, acute pancreatitis, worsening of renal function (creatinine $5.5 \mathrm{mg} / \mathrm{dL}$ ) to oliguria, and marked cognitive impairment. Our case focuses on the importance of an early recognition of laboratory and anamnestic data that may suggest a systemic autoimmune involvement, so as to rapidly undertake an aggressive immunosuppressive therapy.
\end{abstract}

Key words: Systemic lupus erythematous, Acute renal failure, Rhabdomyolysis, Hypoprotidemia

Conflict of interest: None.

Ricevuto: 16 Gennaio 2013; Accettato: 29 Maggio 2013

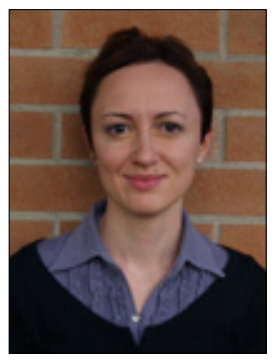

Silvia Forcellini

\section{Introduzione}

Il Lupus Eritematoso Sistemico (SLE) è una connettivite sistemica a eziologia multifattoriale e con patogenesi autoimmune (1).

Nella presentazione più comune si ha il coinvolgimento della cute, delle articolazioni e del sistema muscolo-scheletrico. Sintomi non specifici come astenia, febbricola e iporessia possono creare dif-

LES all'esordio (2). ficoltà e ritardo diagnostico nei casi di

Presentiamo il caso di un paziente affetto da LES, non precedentemente conosciuto, esordito con sintomi gastrointestinali aspecifici e febbre, rapidamente evoluto in insufficienza multiorganica.

\section{Caso clinico}

Un uomo di 51 anni veniva ricoverato in Medicina Interna per la comparsa da circa un mese di epigastralgia e dispepsia. Il paziente riferiva febbricola a domicilio $\left(37-37.5^{\circ} \mathrm{C}\right)$ e sinto- mi aspecifici come astenia, fotofobia, vertigini ed emicrania. Dall'anamnesi risultava una sorella affetta da LES, deceduta "improvvisamente" per insufficienza multiorgano.

All'ingresso: creatininemia $2.5 \mathrm{mg} / \mathrm{dL}$ (GFR $29 \mathrm{~mL} / \mathrm{min}$ con MDRD), ALT 78 U/L, LDH 806 U/L e gamma-GT 229 U/L. All'esame delle urine: proteinuria $400 \mathrm{mg} / \mathrm{dL}$ ed emoglobinuria $1 \mathrm{mg} / \mathrm{dL}$. Il sedimento urinario presentava numerosi globuli rossi e cilindri granulari (Tab. I). Emocolture, urinocolture e coprocolture erano negative. Presente iperpiressia $\left(\mathrm{T} 38.5^{\circ} \mathrm{C}\right)$. All'ecografia addome: dimensioni rene destro $131.5 \mathrm{~mm}$ x 66 $\mathrm{mm}$, rene sinistro $136 \mathrm{~mm} \times 49 \mathrm{~mm}$ e corticale normoecogena e normorappresentata bilateralmente. Nessuna evidenza di dilatazione delle vie biliari e/o di colelitiasi.

Nei giorni successivi, il paziente presentava un progressivo peggioramento della funzione renale (creatininemia $3.8 \mathrm{mg} /$ dL, GFR $21.8 \mathrm{~mL} / \mathrm{min}$ con MDRD) e dopo 12 giorni veniva trasferito alla nostra Unità Operativa.

All'ingresso in U.O. di Nefrologia: T $39.5^{\circ} \mathrm{C}$ e PA $160 / 90$ mmHg. Peso $111 \mathrm{Kg}$ (BMI 33). All'esame obiettivo marcati edemi generalizzati e rallentamento psicomotorio.

Dai primi dati di laboratorio insufficienza renale severa (creatininemia $4.5 \mathrm{mg} / \mathrm{dL})$, anemia $(\mathrm{Hb} 10.9 \mathrm{~g} / \mathrm{dL})$ e rialzo degli indici della citolisi muscolare (CPK $2078 \mathrm{U} / \mathrm{L}$ ). Elettroliti sie- 
TABELLA I - DATI DI LABORATORIO ALL'INGRESSO E DURANTE I PRIMI 10 GIORNI DI RICOVERO NELLA U.O. DI MEDICINA INTERNA

\begin{tabular}{|c|c|c|c|c|c|}
\hline & 10 Set & 11 Set & 13 Set & 15 Set & 20 Set \\
\hline Leucociti (x 1000 u/L) & 5.1 & 3.3 & & & \\
\hline Creatinina (mg/dL) & 2.59 & 2.84 & 2.72 & & 3.86 \\
\hline GFR (mL/min) & 26.2 & 23.6 & 24.8 & & 16.5 \\
\hline LDH (U/L) & 806 & 762 & & & \\
\hline Protidemia (g/dL) & 5.8 & 5.2 & & 4.4 & \\
\hline Albumina $(\%)$ & & & & $52.4 \%$ & \\
\hline PCR (mg/dL) & 1.21 & & & & \\
\hline Esame urine & & $\begin{array}{l}\text { Numerose emazie, } \\
\text { cilindri granulosi }\end{array}$ & & & \\
\hline
\end{tabular}

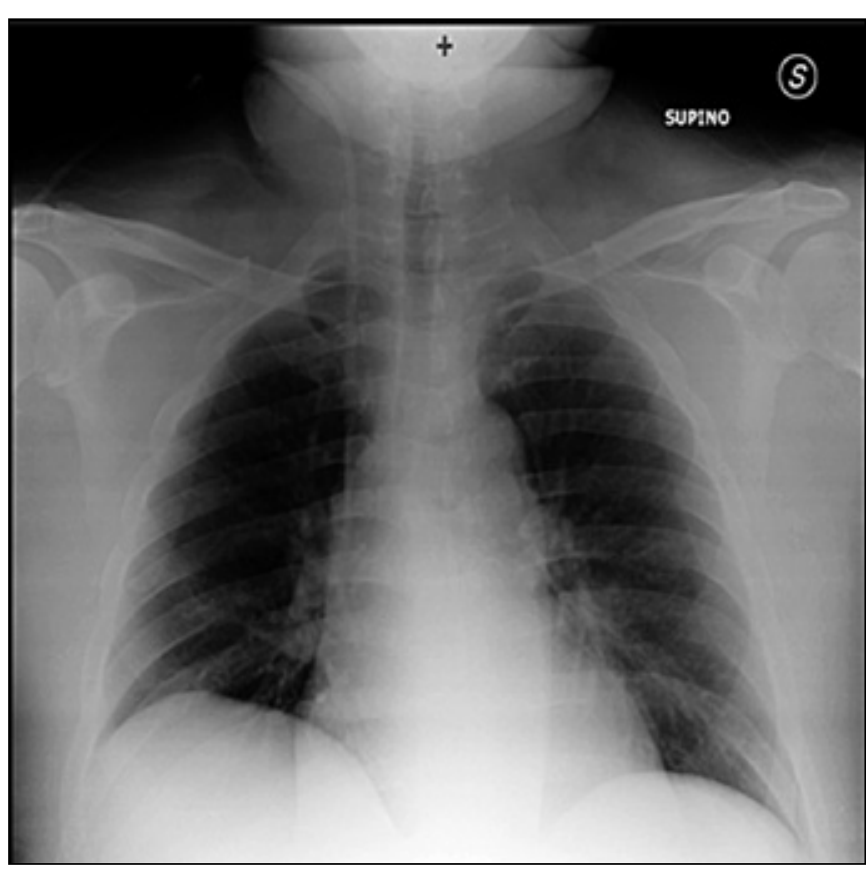

Fig. 1 - RX torace eseguito il giorno precedente il decesso del paziente.

rici: Na $139 \mathrm{mEq} / \mathrm{L}, \mathrm{K} 5.2 \mathrm{mEq} / \mathrm{L}, \mathrm{Ca} 7.6 \mathrm{mg} / \mathrm{dL}$ e P $6.7 \mathrm{mg} /$ dL. Proteinuria ed eritrocituria all'esame delle urine e sedimento urinario "attivo" con globuli rossi dismorfici.

Negativa la ricerca sierologica per infezione recente da Leptospirosi, Toxoplasmosi, Chlamydia, Legionella, Cytomegalovirus, Epstein-Barr virus, Parvovirus B 19 e Herpes virus.

Il pattern immunologico evidenziava: Ab ANA positivi 1:640. $\mathrm{Ab}$ anti Ds-DNA positivi 1:160. Ab anti-Sm 61.3 U.I/mL (v.n $<25$ U.I/mL) e anti-Sm/RNP 80.9 U.I/mL (v.n $<25$ U.I/mL). C3 19 mg/dL (v.n 90-180 mg/dL), C4 3 mg/dL (v.n 16-38 mg/ $\mathrm{dL})$. IgG e $\operatorname{IgM}$ antifosfolipidi negativi.

Durante la degenza, il paziente presentava un rapido peggioramento del quadro rabdomiolitico (creatin chinasi CK $2078 \mathrm{U} /$ $\mathrm{L} \rightarrow 7070 \mathrm{U} / \mathrm{L}$, CK-MB $18.16 \mathrm{ng} / \mathrm{mL} \rightarrow 103 \mathrm{ng} / \mathrm{mL}$, mioglobina $4032 \mathrm{ng} / \mathrm{mL} \rightarrow 11582 \mathrm{ng} / \mathrm{mL}$ e troponinemia $0.31 \mathrm{ng} / \mathrm{mL}$ ), rialzo degli enzimi pancreatici (amilasemia $117 \mathrm{U} / \mathrm{L} \rightarrow 594$ $\mathrm{U} / \mathrm{L}$, lipasemia $175 \mathrm{U} / \mathrm{L} \rightarrow 2132 \mathrm{U} / \mathrm{L}$ ) e incremento di alanina amino transferasi (ALT) $718 \mathrm{U} / \mathrm{L}$. Si riscontravano severa ipoprotidemia $(3.9 \mathrm{~g} / \mathrm{dL})$ e ipoalbuminemia $(1 \mathrm{~g} / \mathrm{dL})$. D-dimero $480 \mathrm{ng} / \mathrm{mL}$ (v.n 0-360 ng/mL).

Sul versante renale si segnalava un ulteriore rialzo della creatininemia $5.3 \mathrm{mg} / \mathrm{dL}$ (GFR $11.7 \mathrm{~mL} / \mathrm{min}$ ), azotemia $370 \mathrm{mg} /$ $\mathrm{dL}$ e oliguria (diuresi sui $400 \mathrm{cc}$ die). Proteinuria delle $24 / \mathrm{h}$ $3.3 \mathrm{~g}$, protidemia $(3.9 \mathrm{~g} / \mathrm{dL})$, albuminemia $(1.6 \mathrm{~g} / \mathrm{dL})$. Profilo elettrolitico: $\mathrm{Na} 140 \mathrm{mEq} / \mathrm{L}, \mathrm{K} 5.6 \mathrm{mEq} / \mathrm{L}, \mathrm{Ca} 7.6 \mathrm{mg} / \mathrm{dL}, \mathrm{P}$ $10.2 \mathrm{mg} / \mathrm{dL}$ (Tab. II).

Indagini strumentali: all'ecocardiografia riscontro di un modesto versamento pericardico e di un "severo collasso del ritorno venoso cavale, come negli stati ipovolemici". Alla RX torace modesti segni di congestione polmonare in assenza di addensamenti polmonari (Fig. 1). ECG: tachicardia sinusale (105 bpm), PR nei limiti, assenza di segni elettrocardiografici di ischemia in atto.

In prima e in seconda giornata, prima del referto del pattern autoanticorpale completo, veniva avviata una terapia con Prednisone $40 \mathrm{mg}$ die ev. In seguito al riscontro di laboratorio di $\mathrm{Ab}$ anti-DNA e anti-Sm (sensibilità 15-30\%, specificità 99\%), marcato consumo del complemento e progressione dell'insufficienza renale, venivano somministrati boli di Metilprednisolone $1 \mathrm{~g}$ die ev per 4 giorni (3). La co-somministrazione di agenti citotossici (Ciclofosfamide) era stata temporaneamente posticipata per la presenza di leucopenia e neutropenia (4).

In settima giornata veniva avviata una terapia sostitutiva con metodica di emofiltrazione isovolemica. A metà seduta, il paziente presentava instabilità emodinamica e collasso cardiocircolatorio. I tentativi di rianimazione praticati risultavano inefficaci e il paziente è deceduto il $20^{\circ}$ giorno complessivo (in Medicina Interna e Nefrologia) di ospedalizzazione.

\section{Discussione}

Il LES può presentare un'ampia gamma di manifestazioni cliniche, da forme "lievi", limitate all'interessamento di cute e articolazioni, a quadri "severi", in caso di coinvolgimento neurologico, renale, ematologico e delle sierose. 
TABELLA II - DATI DI LABORATORIO DURANTE IL PERIODO DI RICOVERO NELLA U.O. DI NEFROLOGIA

\begin{tabular}{|c|c|c|c|c|c|c|c|c|}
\hline & 22 Set & 23 Set & 24 Set & 25 Set & 26 Set & 27 Set & 28 Set & 29 Set \\
\hline $\mathrm{Hb}(\mathrm{g} / \mathrm{dL})$ & 10.9 & 10 & 10.3 & 10.4 & & 11.8 & & 11.7 \\
\hline GB (x 1000) & 2.48 & 2.72 & 2.72 & 2.8 & & 3.1 & & 4.88 \\
\hline Azotemia (mg/dL) & 205 & 203 & 214 & 218 & 245 & 306 & 335 & 370 \\
\hline Creatinina $(\mathrm{mg} / \mathrm{dL})$ & 4.5 & 4.1 & 3.7 & 3.8 & 4.1 & 4.7 & 5.3 & 5.3 \\
\hline Protidemia (g/dL) & & 4.1 & & 3.9 & & & & \\
\hline Albumina (g/dL) & & 1.67 & & 1.51 & & & & \\
\hline Proteinuria 24/h (g) & & & 3.3 & & & 1.3 & 1.8 & \\
\hline ALT (U/L) & 78 & & & & & & & 718 \\
\hline LIPASI (U/L) & 175 & & & & & 388 & 2132 & \\
\hline LDH (U/L) & 1016 & 1031 & 1362 & & & 1877 & & 2455 \\
\hline CPK (U/L) & 2078 & 2525 & 3803 & 4239 & 4895 & 5620 & & 7070 \\
\hline CPK-MB (ng/mL) & 18.16 & & 16.01 & 17.84 & 22.16 & 33.1 & & 103 \\
\hline Mioglobina (mcg/L) & 4032 & 3844 & & 5043 & 6179 & 9965 & 10017 & 11582 \\
\hline Pattern Immunologico & & $\begin{array}{l}\text { ANA } \\
1: 640\end{array}$ & & & $\begin{array}{c}\mathrm{Ab} \text { anti } \\
\text { Ds-DNA } \\
1: 160\end{array}$ & & & $\begin{array}{c}\mathrm{Ab} \text { anti-Sm } \\
61.3\end{array}$ \\
\hline
\end{tabular}

All'ingresso nel nostro reparto, il quadro clinico risultava definito per LES ed erano presenti cinque criteri ARA (5). Sul versante renale, si riscontravano eritrocituria, proteinuria, sedimento urinario "attivo" con globuli rossi dismorfici e insufficienza renale di grado severo. All'emocromo: anemia e leucopenia. All'ecocardiografia vi era un modesto versamento pericardico. Presente un coinvolgimento neurologico con rallentamento psico-motorio. Gli ab-anti-DNA ed ENA (ab anti-Sm) erano positivi, a titolo elevato.

Nel nostro caso, la degenza è stata complicata dalla comparsa di rare, ma potenzialmente fatali complicanze in corso di LES, come la rabdomiolisi e la pancreatite autoimmune. La rabdomiolisi è una sindrome caratterizzata da danno a carico della muscolatura scheletrica con incremento della creatinfosfochinasi di almeno 5 volte rispetto al valore di normalità e l'insorgenza di severe complicanze come insufficienza renale acuta e iperkalemia (6). In letteratura, sono stati descritti solo sei casi di rabdomiolisi correlati a LES (7).

La pancreatite acuta autoimmune è un'altra rara manifestazione nei pazienti affetti da LES (circa 160 casi segnalati) (8), che consegue a deposito di immunocomplessi, attivazione del complemento e insorgenza di vasculite necrotizzante.
Nel nostro caso, l'incremento della lipasemia da $175 \mathrm{U} / \mathrm{L}$ a $2132 \mathrm{U} / \mathrm{L}$ non era riconducibile a colelitiasi e/o a cause esotossiche (9).

Il paziente ha presentato un quadro di nefrite lupica particolarmente severo con rapido declino della funzionalità renale fino all'uremia. L'avvio di una terapia sostitutiva dialitica con metodica di emofiltrazione isovolemica è stata decisa sulla base di oliguria, iperazotemia e comparsa di dispnea dal giorno precedente. Tale scelta è risultata, tuttavia, controversa. I parametri emodinamici sono rimasti, infatti, sostanzialmente stabili durante la degenza e la comparsa di uno shock emodinamico si è verificata nel corso di una seduta emodialitica. Tra le cause del decesso sono stati esclusi eventi cardiovascolari (ECG negativo per eventi ischemici in atto e troponinemia nella norma) e la sindrome da anticorpi antifosfolipidi (anticorpi antifosfolipidi negativi). La RX torace non suggeriva complicanze pneumologiche possibili in quadri di LES severo come polmoniti, fibrosi interstiziale ed emorragie polmonari $(10,11)$. L'ipotesi di embolia polmonare risultava improbabile per valori di D dimero solo modicamente aumentati rispetto alla normalità.

Il riscontro di edemi, marcata ipoalbuminemia e ipoproti- 
demia e severo collasso del ritorno venoso cavale all'ecocardiografia supporta l'ipotesi che, alla base dello shock emodinamico, ci sia stato lo sviluppo di un'ipovolemia conseguente alla concomitanza di rabdomiolisi, pancreatite acuta e nefrite lupica (formazione del "terzo spazio").

Nel nostro caso, la sintomatologia gastrointestinale associata a iperpiressia $\left(\mathrm{T} 39^{\circ} \mathrm{C}\right.$ ), in un caso di LES non conosciuto, ha indotto, all'ingresso, a ipotizzare un'origine gastroenteritica infettiva. Boli di metilprednisolone da $1 \mathrm{~g}$ die sono stati, quindi, avviati solo nei cinque giorni precedenti l'exitus, quando erano già comparsi i segni, oltre che della nefrite acuta, anche di rabdomiolisi, pancreatite acuta e coinvolgimento neurologico. La prosecuzione con ciclofosfamide ev e l'esecuzione della biopsia renale erano state programmate a conclusione del ciclo della terapia cortisonica.

\section{Conclusioni}

Il LES può presentarsi all'esordio con quadri “severi”, quando coesistono i coinvolgimenti neurologico, renale, ematologico e delle sierose.

Il nostro caso sottolinea l'importanza di un rapido riconoscimento di dati anamnestici e laboratoristici sospetti di LES "grave", in particolare nelle forme non conosciute. La possibilità di una rapida evoluzione verso l'insufficienza multiorgano richiede, quindi, l'avvio tempestivo e aggressivo di una terapia immunosoppressiva prima dell'insorgenza di complicanze sistemiche potenzialmente fatali.

\section{Riassunto}

Il Lupus Eritematoso Sistemico (LES) è una connettivite autoimmune sistemica che coinvolge più frequentemente la cute e il sistema muscolo-scheletrico e articolare. Quadri di LES "grave" all'esordio possono presentarsi con manifestazioni cliniche aspecifiche ed evolvere rapidamente verso l'insufficienza multiorgano. Abbiamo riportato il caso di un paziente affetto da LES sistemico, non precedentemente diagnosticato, esordito con sintomatologia gastrointestinale e iperpiressia. I dati di laboratorio all'ingresso segnalavano insufficienza renale acuta, proteinuria ed emoglobinuria. L'evoluzione rapida e infausta verso l'insufficienza multiorgano e l'exitus (venti giorni) sottolineano l'importanza di riconoscere tempestivamente segni clinici e di laboratorio suggestivi per LES "grave", in modo da avviare il più precocemente possibile una terapia immunosoppressiva specifica.

Parole chiave: Lupus Eritematoso Sistemico, Insufficienza Renale Acuta, Rabdomiolisi

Dichiarazione di conflitto di interesse: Gli Autori dichiarano di non avere conflitto di interessi.

Indirizzo degli Autori:

Dr.ssa Silvia Forcellini

U.O. di Nefrologia e Dialisi

Azienda Ospedaliero Universitaria Sant'Anna Cona

Via A. Moro 8

44100 Ferrara

silviaforcellini@gmail.com

\section{Bibliografia}

1. Mills JA. Systemic lupus erythematosus. N Engl J Med 1994; 330: 1871-9.

2. Wallace DJ. The clinical presentation of systemic lupus erithematosus. In: Wallace DJ, Hahn BH, editors. Dubois' lupus erythematosus. 5th ed. Baltimore: Lippincott Williams\&Wilkins: 1997; 630.

3. Parker BJ, Bruce IN. High dose methylprednisolone therapy for the treatment of severe systemic lupus erythematosus. Lupus 2007; 16 (6): 387-93.

4. Bargman JM. How did cyclophosphamide become the drug of choice for lupus nephritis? Nephrol Dial Transplant 2009; 24: 381-4.

5. Tan EM, Cohen AS, Fries JF, et al. The 1982 revised criteria for the classification of systemic lupus erithematosus. Arthritis Rheum 1982; 25: 1271-7.

6. Singh D, Chander V, Chopra K. Rhabdomyolysis. Methods Find
Exp Clin Pharmacol 2005; 27: 39-48.

7. de Carvalho JF, da Mota LM, Bonfa E. Fatal Rhabdomyolysis in systemic lupus erythematosus. Rheumatol Int 2011; 31: 1243-5.

8. Eaker EY, Toskes PP. Systemic lupus erithematosus presenting initially with acute pancreatitis and a review of the literature. Am J Med Sci 1989; 297: 38-41.

9. Tian XP, Zhang X. Gastrointestinal involvement in systemic lupus erythematosus: insight into pathogenesis, diagnosis and treatment. World J Gastroenterol 2010; 16 (24): 2971-7.

10. Ghosh A, Das T, Ghosh A, Karmakar P, Pal J. Evaluation of respiratory manifestations in systemic lupus erythematosus with special reference to pulmonary interstitial involvement. J Indian Med Assoc 2012; 110 (2): 109-11.

11. Guidelines for referral and management of systemic lupus erythematosus in adults. American College of Rheumatology Ad Hoc Committee on Systemic Lupus Erythematosus Guidelines. Arthritis Rheum 1999; 42 (9): 1785-96. 


\section{TEST di VERIFICA}

1) Quale tra le seguenti manifestazioni cliniche e di laboratorio NON è di frequente riscontro nel LES?

a) Anemia normocitica, normocromica

b) Proteinuria ed eritrocituria

c) Pancreatite acuta autoimmune

d) Coinvolgimento cutaneo e articolare

e) Leucopenia

2) L'anticorpo anti-SM:

a) Ha bassa sensibilità e alta specificità

b) Ha elevata sensibilità ma bassa specificità

c) Ha elevata sensibilità

d) Ha bassa specificità

e) Ha alta sensibilità e alta specificità

3) Quale manifestazione clinica NON è stata descritta nel nostro caso?
a) Rabdomiolisi
b) Pancreatite autoimmune
c) Insufficienza renale acuta
d) Rash malare
e) Coinvolgimento neurologico con rallentamento psicomotorio

Le risposte corrette alle domande sono pubblicate su questo numero del Giornale di Tecniche Nefrologiche \& Dialitiche Vol. 25, no. 2, pag. 117. 